Z. Klin. Chem. Klin. Biochem.

11. Ig. 1973, S. $381-387$

\title{
Verbesserung und Qualitätskontrolle der radioimmunologischen Thyreotropin-Bestimmung1)
}

\author{
Von F. Erhardt, I. Marschner, Renate C. Pickardt und P. C. Scriba
}

Aus der II. Medizinischen Universitätsklinik (Direktor: Prof. Dr. E. Bucbborn) München

(Eingegangen am 15. März/7. Juni 1973)

Durch Messung der Reaktionskinetiken von ${ }^{125} \mathrm{~J}-\mathrm{TSH}$ mit Anti-H-TSH bei verschiedenen Temperaturen wurde die Inkubation bei Raumtemperatur als optimal erkannt. Die Präzipitationsreaktion mit Anti- $\gamma$-Globulin erfolgt bei den meisten der geprüften Chargen bei Raumtemperatur komplett innerhalb weniger Stunden. Die Anwendung von niedrig markiertem ${ }^{125} \mathrm{~J}-\mathrm{TSH}(20-70 \mathrm{mCi} / \mathrm{mg})$ führt wegen der gut erhaltenen Immunoreaktivität des Tracers zu empfindlichen Eichkurven, und im Vergleich zu Wiederfindekurven in Humanserum richtigen TSH-Werten. Durch Wiederfindekurven in Serum erreichen wir einen Interassay-Variationskoeffizienten von etwa 10 Prozent und verbessern dadurch die relative Richtigkeit der gefundenen TSH-Werte.

\section{Improvement and quality control of the radioimmunological TSH determination}

The reaction kinetics of ${ }^{125}$ I-TSH with Anti-H-TSH were studied at different temperatures, and the incubation was found to be optimal at room temperature. The precipitation reaction with anti- $\gamma$-globulin is complete in most of the cases investigated within a few hours. The use of low labelled ${ }^{125} \mathrm{I}$-TSH $(20-70 \mathrm{mCi} / \mathrm{mg})$ leads to higher sensitivity, because of the scarcely altered immunoreactivity of the tracer, and, with comparison of recovery curves in human serum, to more accurate TSH values. Recovery curves in serum reduce the interassay variation coefficient to about $10 \%$ and improve the relative accuracy of the TSH values found.

Seit Utiger, Odell und CONDLIFFe 1963 zum ersten $\mathrm{Mal}$ die radioimmunologische Bestimmung von $\mathrm{H}$ $\mathrm{TSH}^{2}$ ) beschrieben (1), sind eine Reihe methodischer Arbeiten erschienen, in denen durch Tracer-Nachreinigung (2), Verwendung von H-TSH-armen Humanseren für die Standardkurven $(3,4)$ und Einsatz sehr niedriger Mengen von hoch markiertem H-TSH $(3,5)$ Empfindlichkeit und Richtigkeit der TSH-Bestimmung gesteigert werden. Die Vorinkubation wird nicht von allen Autoren als wirksames Mittel zur Steigerung der Empfindlichkeit anerkannt (5). Eine Inkubationszeit von 5 bis 7 Tagen bei $4^{\circ} \mathrm{C}$ zur Erreichung des Reaktionsgleichgewichtes ist die Regel. Wir haben einige Parameter der radioimmunologischen H-TSH-Bestimmung systematisch untersucht, um optimale Reaktionsbedingungen zu erhalten und zugleich einen Vorschlag zur relativen Richtigkeitskontrolle erarbeitet.

\section{Material und Methoden}

Puffer (alle $\mathrm{pH}$ 7.4)

Puffer A-Stammlösung: 10-fache Konzentration: $0,15 \mathrm{~mol} / \mathrm{l}$ Phosphat, 1,5 mol/1 NaCl, $2 \mathrm{~g}$ Merthyolat/1, $20 \mathrm{~g}$ Rinderalbumin/l (Pentex, Fluka, Buchs, Schweiz), 0,1 mol/1 EDTA.

Puffer A-Gebrauchslösung: Stammlösung mit dest. Wasser 1:10 verdünnen.

1) Mit Unterstützung dex Deutschen Forschungsgemeinschaft (SFB 51).

2) Abkürzungen: H-TSH = humanes Thyrcotropin; HCG = humanes Choriongonadotropin; $T=$ Gesamtradioaktivität per Probe; $\mathrm{B}=$ gebundene Radioaktivität; $\mathrm{B}_{0}=$ Standard-Leerwert; $\mathbf{N}=$ unspezifische Bindung ( + Nullrate des Gammazählers); $\mathrm{K}_{\text {niedrig }}=\mathrm{TSH}-$ Werte des Kontrollserums mit niedrigem TSHGehalt; $K_{\text {noch }}=$ TSH-Werte des Kontrollserums mit hohem TSH-Gehalt; AK = Antikörper; TRH = Thyreotropin Releasing Hormone.
Puffer B: Puffer A ohne EDTA, dazu $40 \mathrm{~g}$ Rinderalbumin (Pentex) auf 11 Gebrauchslösung.

Puffer C: $0,15 \mathrm{~mol} / 1 \mathrm{NaCl}, 0,015 \mathrm{~mol} / 1$ Natriumphosphatpuffer. Puffer D: $0,4 \mathrm{~mol} / 1$ Natriumphosphatpuffer.

AK1-Lösung $=$ Anti-H-TSH (für 100 Proben)

$50 \mu \mathrm{l}$ Anti-H-TSH 1:100 (in dieser Konzentration eingefroren gelagert (NIAMD, Bethesda)), 1000 E HCG (Primogonyl, Schering AG, Berlin), $40 \mu$ l Kaninchen-Normalserum, ad $60 \mathrm{ml}$ mit Puffer A auffüllen.

Für die Messung der unspezifischen Bindung (N) benötigt man die Lösung ohne Anti-TSH (Tab. 1).

AK2-Lösung $=Z_{\text {weiter Antikörper (präzipitierender Antikörper) }}$ Anti-Rabbit-Precipitating-Serum, Donkey, Fa. Wellcome, Lot. K 3437 1:50 oder Lot. K 6042 1:25 mit Puffer A verdünnt. Bei allen neuen Chargen muß das Optimum der Verdünnung und der Inkubationszeit neu festgestellt werden.

\section{TSH-Standard}

Von einer H-TSH-Stammlösung (Human Pituitary TSH 68/38, NIMR, Mill Hill, London) mit $10 \mathrm{mE} / \mathrm{ml}$ werden $0,2 \mathrm{ml}$ mit Puffer B auf $20 \mathrm{ml}$ aufgefüllt (Standardlösung $100 \mu \mathrm{E} / \mathrm{ml}$ ) und eine Verdünnungsreihe erstellt (Abb. 3), die in Portionen zu $0,8 \mathrm{ml}$ eingefroren wird.

Durch Zufügen von H-TSH-Standard zu Serum eines Patienten, dessen eigener TSH-Spiegel durch $\mathrm{T}_{3}$-Behandlung $(100 \mu \mathrm{g} / \mathrm{Tag})$ supprimiert ist, stellt man sich eine Wiederfindekurve (Abb. 6) her.

TSH-Markierung mit ${ }^{125}$ Jod nach GreENwood et al (6)

mit $\mathrm{Na}^{125} \mathrm{~J}$ zur Proteinmarkierung (Farbwerke Hoechst AG, Frankfurt/Main oder The Radiochemical Centre Amersham, England).

$2,5 \mu \mathrm{g}$ TSH (NIAMD, Bethesda) werden mit $0,5 \mathrm{mCi}{ }^{125} \mathrm{~J}, 30 \mu \mathrm{g}$ Chloramin $\mathrm{T}$ und $20 \mu \mathrm{l}$ Puffer $\mathrm{D}$ in einem Gesamtvolumen von $40 \mu 15$ bis $15 \mathrm{~s}$ geschüttelt. Die Reaktion wird mit $60 \mu \mathrm{g}$ Natriummetabisulfit in $20 \mu \mathrm{l}$ Puffer $\mathrm{C}$ gestoppt, das Gemisch auf die Säule gebracht und das Reaktionsgefäß mit $500 \mu \mathrm{g} \mathrm{KJ}$ in $50 \mu \mathrm{l}$ dest. Wasser nachgespült. 
Tab. 1

Pipettier- und Inkubationsschema

\begin{tabular}{lccc}
\hline . & Probe & $\mathrm{N}$ & . Leerwert \\
\hline 1. Tag & & & \\
Serum bzw. Standard & $200 \mu l$ & - & - \\
Puffer B & - & $200 \mu l$ & $200 \mu l$ \\
AK 1-Lösung mit Anti-TSH & $600 \mu l$ & - & $600 \mu l$ \\
AK 1-Lösung ohne Anti-TSH & & $600 \mu l$ & \\
\hline
\end{tabular}

mit Dilutor* zusammen pipettieren (gute Durchmischung) und etwa $44 \mathrm{~h}$ bei $18-25^{\circ} \mathrm{C}$ (Raumtemperatur) inkubieren. Verschluß der deckellosen Eppendorfgefäße während der Inkubationszeiten mit Selbstklebefolie.

\section{Tag}

$128 \mathrm{~J}-\mathrm{TSH}$ (Tracer)

überall $100 \mu l$

mit Hamilton-Spritze zufügen (gute Durchmischung), anschließend etwa $60 \mathrm{~h}$ bei Raumtemperatur inkubieren.

6. Tag
AK 2-Lösung überall $200 \mu \mathrm{l}$

mit Hamilton-Spritze zufügen, anschließend $3 \mathrm{~h}$ bei Raumtemperatur inkubieren. Von etwa zehn Proben wird die Gesamtaktivität gemessen. Dann werden sämtliche Proben 5 min lang bei $6000 \mathrm{~g}$ zentrifugiert, die Überstände (automatisch**) abgesaugt und die Rückstände $1 \mathrm{~min}$ im Gamma-Zählgerät*** gezählt.

* Fisons-Diluter, iFa. Ima, München.

** Apparatur unter Verwendung einer 25 Kanal-Mikropumpe (Ismatec, Zürich), die dankenswerterweise von Herrn Dr. Ing. H. SchWAB, SFB 51, München, gebaut wurde.

*** Gamma-Zählgerät: Gammaguard, Tracerlab.

Die Isolierung des Tracers erfolgt durch Säulenchromatographie an albumindesaktiviertem Sephadex G $75(8 \times 150$ bis $200 \mathrm{~mm})$. Die TSH-Fraktion mit der höchsten Aktivität und die beiden folgenden werden sogleich auf etwa $250000 \mathrm{Imp}$./min pro $\mathrm{ml} \mathrm{mit}$ Puffer $\mathrm{A}$ verdünnt und bei $-30^{\circ} \mathrm{C}$ eingefroren. Die Lösung kann bis zu drei Wochen lang verwendet werden, wenn die spezifische Aktivität $70 \mathrm{mCi} / \mathrm{mg}$ TSH nicht übersteigt.

Die Berechnung der spezifischen Aktivität erfolgt aus eingesetzter TSH- und ${ }^{125} \mathrm{~J}$-Menge (unter Zugrundelegung der Aktivitätsangaben des Isotopen-Herstellers) und aus dem Aktivitätsverbältnis von TSH- und Jodid-Fraktion nach der Säulenchtomatographie.

\section{Metbode (Tab. 1)}

In jeder Serie laufen folgende Proben neben den Serumwerten mit:

1. Sechs Proben ohne Antikörper zur Messung der unspezifischen Bindung $\mathrm{N}$.

2. Je drei Proben von zwei Kontrollseren mit einem niedrigen TSH-Gehalt $(2-5 \mu \mathrm{E} / \mathrm{ml})$ und einem höheren Wert (etwa $25 \mu \mathrm{E} /$ $\mathrm{ml}$ ) am Anfang und am Ende eines Durchgangs.

3. Zehn Punkte der Standardkurve in Dreifachwerten.

4. Neun Punkte einer Wiederfindekurve von TSH-Standard in $\mathrm{T}_{3}$-supprimiertem Patienten-Serum in Dreifachwerten.

5. Neun Einzelwerte des Standard-Leerwertes zur Berechnung der unteren Nachweisgrenze.

\section{Auswertung}

Nach Abzug der unspezifischen Bindung (N) wird die Impulszahl des Standard-Lecrwerts gleich $100 \%$ gesetzt $\left(B_{0}\right)$. Man erhält eine Eichkurve mit der Abszisse $\mu \mathrm{E} / \mathrm{ml}$ und mit der Ordinate $\frac{\mathrm{B}-\mathrm{N}}{\mathrm{B}_{0}-\mathrm{N}} \times 100[\%]$.

\section{Ergebnisse}

Temperaturabhängigkeit und Inkubationszeiten

Kinetik der Bindung von 125J-TSH an Anti-TSH-Serum bei verscbiedenen Temperaturen

Die Kinetiken (Abb. 1a) zeigen, daß das Gleichgewicht auch nach $140 \mathrm{~h}$ bei keiner Temperatur ganz erreicht wird, daß die Reaktion bei $20^{\circ} \mathrm{C}$ doppelt so schnell verläuft wie bei $4^{\circ} \mathrm{C}$ und daß die höchste Bindung bei $20^{\circ} \mathrm{C}$ erreicht wird. Die unspezifische Bindung bleibt bei allen Temperaturen bei 2,5\% der Gesamtaktivität konstant.

Abbildung $1 \mathrm{~b}$ zeigt, da $\mathrm{B}$ die niedrige Lage des $37^{\circ} \mathrm{C}$ Plateaus bei $22^{\circ} \mathrm{C}$ in Richtung geringerer Dissoziation verschoben wird, doch wird das $22^{\circ} \mathrm{C}$-Plateau nicht ganz

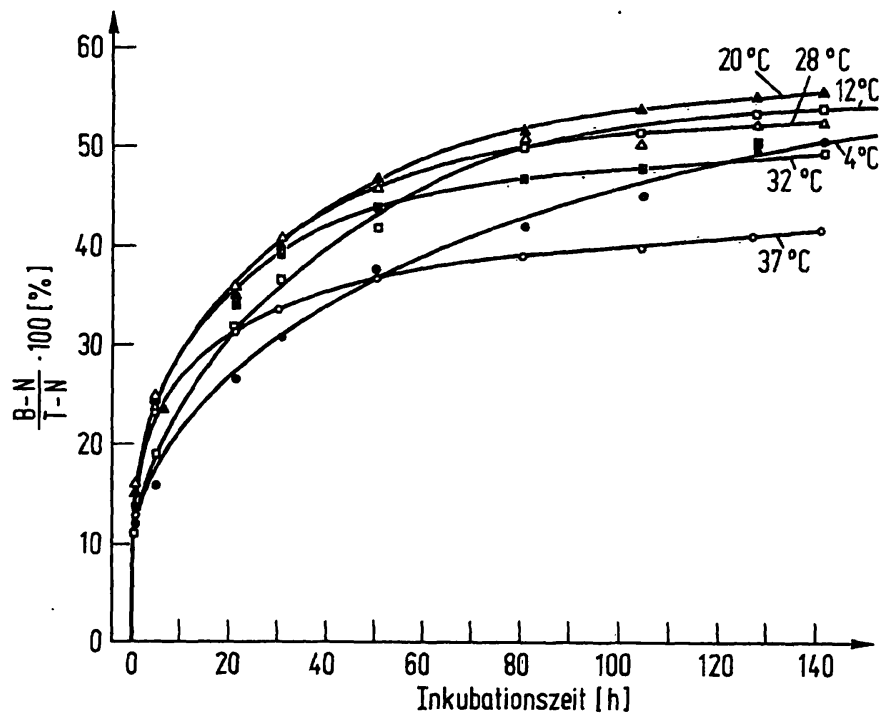

Abb. 1 a

Kinetik der Bindung von ${ }^{125} \mathrm{~J}-\mathrm{TSH}$ an Anti-TSH-Serum bei verschieIm Kühlraum bei $+4^{\circ} \mathrm{C}$ wurden Temperaturen

Im Puffer B, 0,1 ml ${ }^{125} \mathrm{~J}-\mathrm{TSH}$ (spezifische Aktivität $112 \mathrm{mCi} / \mathrm{mg}, 25000$ Imp./min, $450 \mathrm{pg}$ ) und $0,6 \mathrm{ml}$ Anti- TSH pipettiert.

Je 20 Proben wurden dann bei $4,20,28,32$ und $37^{\circ} \mathrm{C}$ gleichzeitig inkubiert. In den angegebenen Zeitintervallen, zuletzt nach $140 \mathrm{~h}$ wurden je 2 Proben entnommen und bei $-30^{\circ} \mathrm{C}$ eingefroren. Sämtlich Proben wurden gleichzeitig 3 Minuten lang bei $28^{\circ} \mathrm{C}$ aufgetaut und mit je $0,2 \mathrm{ml} \mathrm{AK} 2$-Lösung versetzt

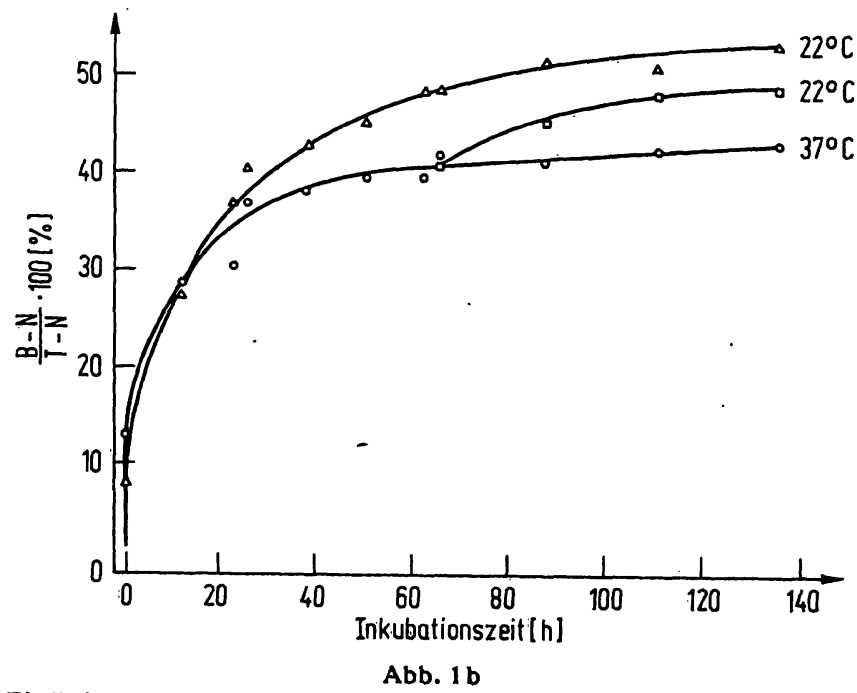

Einfluß der Dissoziation auf die Bindung von ${ }^{128} \mathrm{~J}-\mathrm{TSH}$ bei $37^{\circ} \mathrm{C}$ Ansatz wie Abb. $1 \mathrm{a}$. Nach 65 ih wurden 4 Doppelproben von $37^{\circ} \mathrm{C}$ nach $22^{\circ} \mathrm{C}$ überführt 


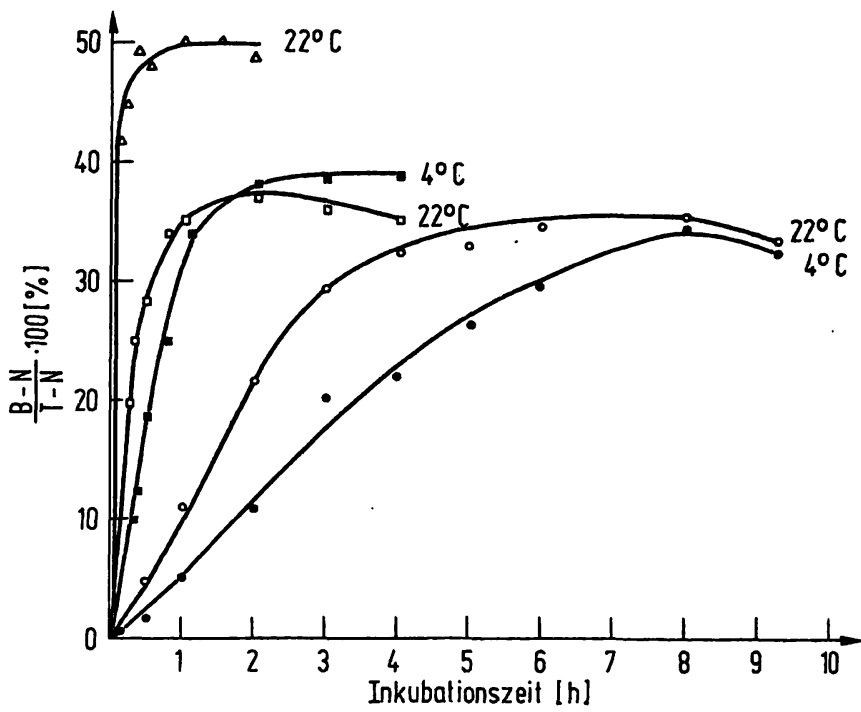

Abb. 2

Kinetiken der Fällungsreaktion mit Anti- $\gamma$-Globulinen verschiedener Chargen bei Raumtemperatur und bei $4^{\circ} \mathrm{C}$

20 Proben mit $0,2 \mathrm{ml}$ Puffer $B$ wurden $48 \mathrm{~h}$ lang mit $0,1 \mathrm{ml}$ AK 2-Lösung optimaler Konsuntration abständen wurden je 2 Proben entnommen und sofort zentrifugiert $\Delta-\Delta$ (Wellcome Lot $K 3437$, Verdünnung $1: 50$ )

-

0 - (Antibody Incorp. Lot 11.16.72, Verdünnung 1:64)

èrreicht. Das bedeutet, daß ${ }^{125} \mathrm{~J}$-TSH bei $37^{\circ} \mathrm{C}$ in $65 \mathrm{~h}$ auch immunologische Aktivität verliert. Die niedrigere Lage des $37^{\circ} \mathrm{C}$-Plateaus (Abb. $1 \mathrm{a}$ und $1 \mathrm{~b}$ ) setzt sich also aus Dissoziationsgleichgewicht und „Damage“ zusammen.

\section{Kinetiken der Fällungsreaktion mit Anti-y-Globulinen verschiedener Chargen}

Die Präzipitation mit 2. Antikörper bei Raumtemperatur dauert je nach Charge zwischen $5 \mathrm{~min}$ und einigen Stunden. Bei $4^{\circ} \mathrm{C}$ erfolgt die Präzipitation im allgemeinen wesentlich langsamer (Abb. 2).

Nicht zuletzt aus Gründen der Wirtschaftlichkeit lohnt es sich, das günstigste Verhältnis zwischen KaninchenNormalserum und AK 2-Verdünnung auszutesten. Abbildung 3 zeigt, da $\beta$ man durch Verwendung von nur 0,2 $\mu \mathrm{l}$ Kaninchen-Serum per Probe mit der Hälfte des teuren 2. Antikörpers auskommt, ohne dadurch die Menge an gefällter Aktivität merklich zu verringern.

\section{Einfluß der Vorinkubation auf die Empfindlicbleeit}

Abbildung 4 zeigt vier Standardkurven verschiedener Steilheit, sowie deren untere Nachweisgrenzen. Diese berechnen wir aus der Streuung des Standardleerwertes, indem wir den dreifachen Variationskoeffizienten von $B_{0}\left(B_{0}=100 \%\right)$ abziehen und den entsprechenden TSH-Wert auf der Standardkurve ablesen.

Diese liegen in dieser Versuchsserie nach heißer Vorinkubation bei $10 \mu \mathrm{E} \mathrm{TSH} / \mathrm{ml}$ (A), nach gleichzeitiger Inkubation bei $2 \mu \mathrm{E} \mathrm{TSH} / \mathrm{ml}$ (B), nach $44 \mathrm{~h}$ kalter Vorinkubation bei $0,7 \mu \mathrm{E}$ TSH/ml (C) und nach $140 \mathrm{~h}$ kalter Vorinkubation bei $0,4 \mu \mathrm{E} \mathrm{TSH} / \mathrm{ml}$ (D).

Die Vorinkubation mit kaltem Hormon steigert die Empfindlichkeit also bedeutend. Es muß betont werden, $\mathrm{da} B$ die spezifische Aktivität des eingesetzten Tracers

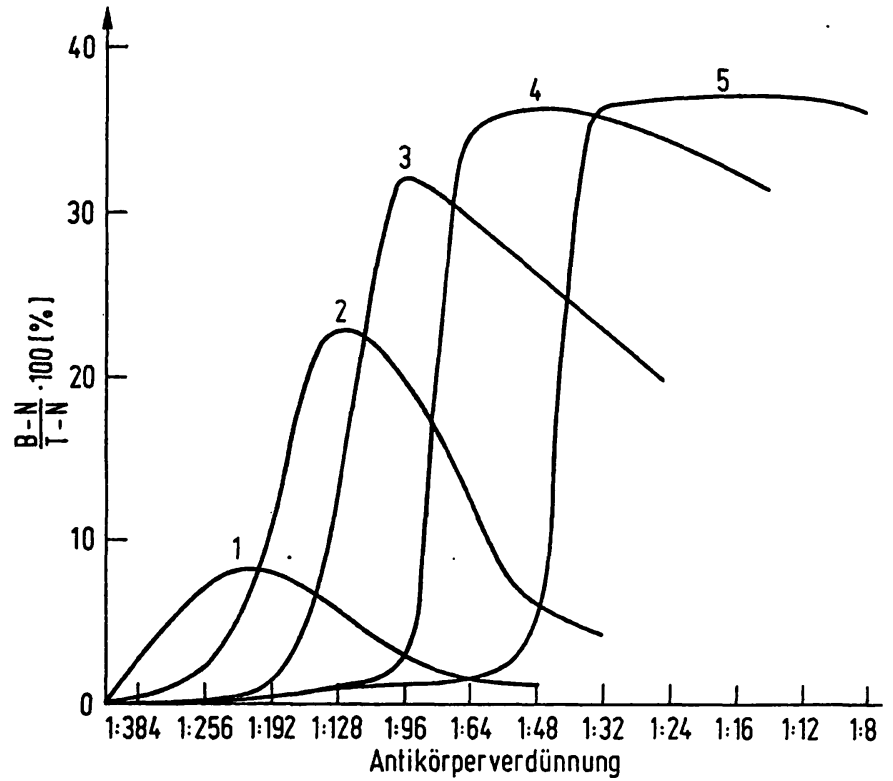

Abb. 3

Optimierung der Fällung mit Anti-Kaninchen- Präzipitationsserum (Wellcome: Lot 6042)

Kurven 1 bis 5 unterscheiden sich durch die Mengen Normal-Kaninchen-

$(1=0,025 \mu 1 ; 2=0,05 \mu 1 ; 3=0,1 \mu 1 ; 4=0,2 \mu 1 ; 5=0,4 \mu l)$

Abszisse: Antiserum-Verdünnung (davon $0,1 \mathrm{ml}$ per Probe)

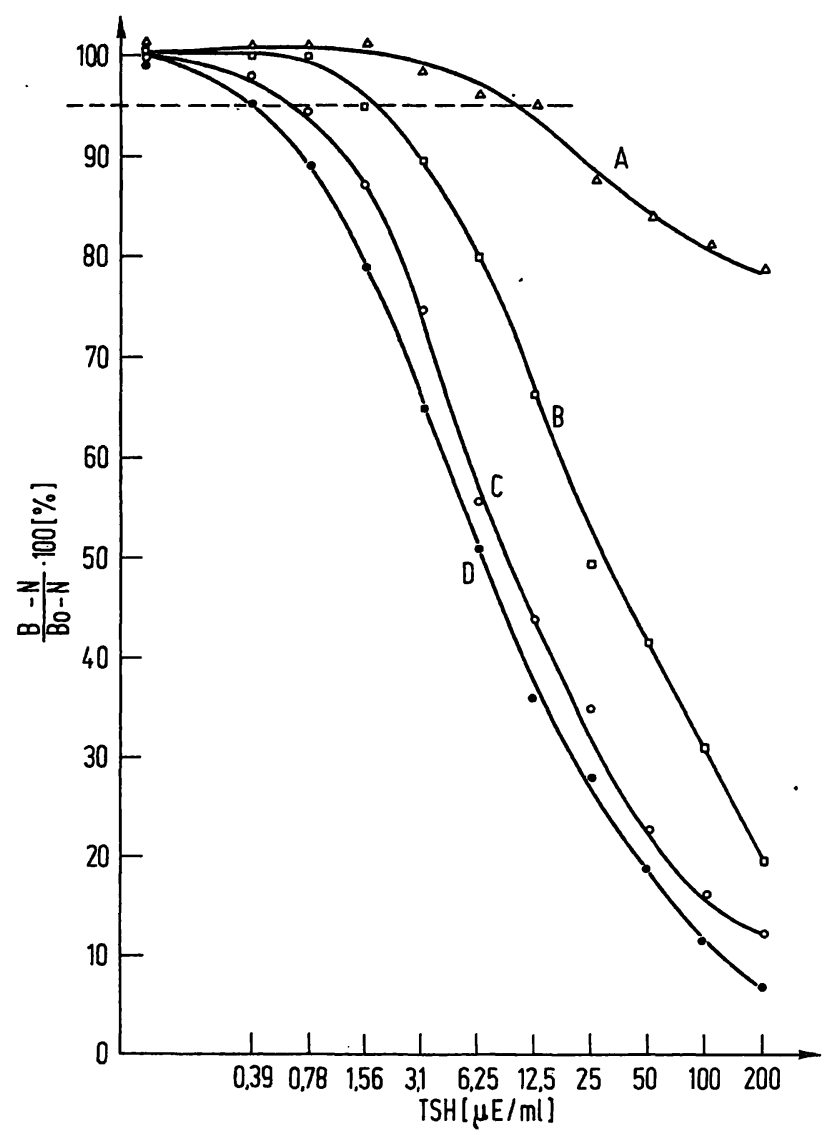

Abb. 4

Einfluß der Vorinkubation auf Standardkurven in Puffer $B$ Spezifische Aktivität des ${ }^{181} \mathrm{~J}-\mathrm{TSH}=36 \mathrm{mCi} / \mathrm{mg} ; 22600 \mathrm{Imp} . / \mathrm{min}$ per A: $44 \mathrm{~h}$ Vorinkubation mit Tracer und Antikörper 1; anschließend $63 \mathrm{~h}$ Inkubation mit Standard $(\Delta-\Delta)$

B: $63 \mathrm{~h}$ gleichzeitige Inkubation von Tracer, Standard und Anti-

C: $44 \mathrm{~h}$ Vorinkubation von Standard und Antikörper 1; anschließend

$63 \mathrm{~h}$ mit Tracer ( $O-O)$ ( ) $140 \mathrm{~h}$ Vorinkubation mit Standard und Antikörper 1; anschließend $63 \mathrm{~h} \mathrm{mit} \mathrm{Tracer}(0-0)$

Die gestrichelte Linie zeigt die untere Nachweisgrenze für alle 4 Kurven 
nur $36 \mathrm{mCi} / \mathrm{mg}$ betrug, entsprechend $\sim 1250 \mathrm{pg}$ TSH pro Probe.

Einfluß der spezifischen Aktivität des ${ }^{12.5} \mathrm{~J}$-TSH auf die Haltbarkeit des Tracers

Eine Charge mit $5 \mathrm{mCi} \mathrm{Na}^{125} \mathrm{~J}$ (Angabe des Herstellers) wurde in Portionen zu $1 \mathrm{mCi}$ aufgeteilt und durch Variation der Reaktionsbedingungen ${ }^{125} \mathrm{~J}$-TSH mit spezifischen Aktivitäten von 43, 123 und $220 \mathrm{mCi} / \mathrm{mg}$ hergestellt. Aliquots der Tracer wurden anschließend über $4 \mathrm{~g}$ Cellulose in Säulen von $15 \times 50 \mathrm{~mm}$ (Avicel, Merck) nachgereinigt und die ${ }^{125} \mathrm{~J}-\mathrm{TSH}$ enthaltenden Fraktionen auf $360000 \mathrm{Imp} . / \mathrm{min} \cdot \mathrm{ml}$ verdünnt. Der Rest wurde unverdünnt und ohne weitere Nachreinigung über Cellulose bei $-30^{\circ} \mathrm{C}$ eingefroren. Um eine zu hohe Markierung des TSH zu vermeiden, markieren wir üblicherweise $2,5 \mu \mathrm{g}$ TSH mit $0,5 \mathrm{mCi}$ $\mathrm{Na}^{125} \mathrm{~J}$ und Chloramin $\mathrm{T} 5-15 \mathrm{~s}$ lang. Wir haben die Konzentrationsabhängigkeit der Zersetzungsreaktion verschieden hoch markierter Tracer untersucht. Dazu wurde frisch markiertes TSH auf Gebrauchskonzentration verdünnt und in konzentriertem Zustand (unverdünnte Fraktionen nach Sephadex-Chromatographie des Markierungs̀gemisches) bei $-30^{\circ} \mathrm{C}$ eingefroren. Nach zwei Wochen wurde das Material durch Rechromatographie über kurze Sephadex G 75-Säulen (15 X $75 \mathrm{~mm}$ ) untersucht.

Tabelle 2 zeigt, daß sich das Verhältnis von ${ }^{125} \mathrm{~J}$-TSHPeak zu Jodid-Peak bei konzentrierter Aufbewahrung zu Ungunsten des J-TSH-Anteils verändert, während der Jodid-Anteil schneller zunimmt. Niedrig markierter Tracer ( $43 \mathrm{mCi} / \mathrm{mg}$ ) spaltet bei gleicher Aktivität per Volumeneinheit $(360000 \mathrm{Imp} . / \mathrm{min} \cdot \mathrm{ml}$ ) nur $12 \%$ Jodid in 14 Tagen $\mathrm{ab}$, weshalb der Tracer unter unseren Bedingungen drei Wochen lang ohne Nachreinigung verwendbar bleibt.

Mit den 3 Tracern verschiedener spezifischer Aktivität (Abb. 5) wurden Standardkurven in Puffer B und in TSH-armem Serum simultan angesetzt. Das mit $43 \mathrm{mCi} /$ mg niedrig markierte TSH mit einem $\frac{B_{0}}{T}$ von $42 \%$ wird wesentlich besser gebunden als drei- und fünffach so hoch markiertes TSH (123 und $220 \mathrm{mCi} / \mathrm{mg}$ ), obwohl bei gleicher Aktivität nur etwa $1 / 3$ bzw. $1 / 5$ der ${ }^{125} \mathrm{~J}$ TSH-Menge per Probe eingesetzt wurde. Dies zeigt, $\mathrm{da} ß$ die Immunoaffinität mit zunehmender Markierung abnimmt.

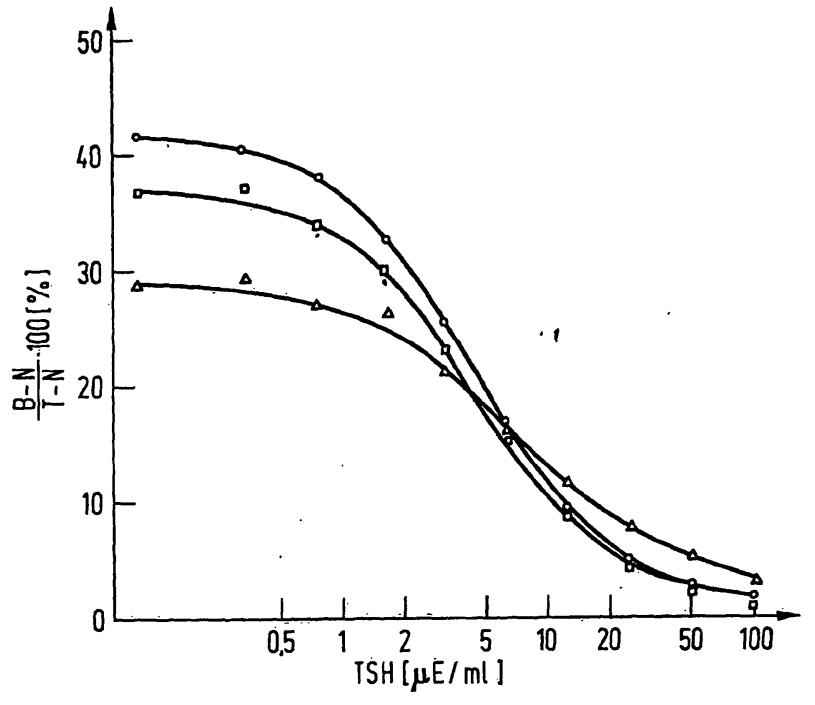

Abb. 5 .

Standardkurven in Puffer B mit ${ }^{125} \mathrm{~J}$-TSH bei verschiedener spezifischer

Spezifische Aktivitäten: $43 \mathrm{mCi} / \mathrm{mg}(O-O), 123 \mathrm{mCi} / \mathrm{mg}(\square-\square)$, $220 \mathrm{mCi} / \mathrm{mg}(\triangle-\Delta)$

Bedeutung der spezifischen Aktivität für Empfindlichkeit und Richtigkeit der TSHBestimmung

Abbildung 6 zeigt Standardkurven in Puffer B und Wiederfindekurven in TSH-armem Serum. Die Standardkurve mit dem niedrigst markierten Tracer $(43 \mathrm{mCi} /$ $\mathrm{mg}$ ) und die mit dem $z$ weiten Tracer $(123 \mathrm{mCi} / \mathrm{mg})$ sind in $\frac{\mathrm{B}}{\mathrm{B}_{0}}$-Auftragung identisch. Die Standardkurve mit dem höchst-markierten Tracer $(220 \mathrm{mCi} / \mathrm{mg})$ verläuft nach rechts versetzt, d. h., der Assay wäre weniger empfindlich. Die Wiederfindekurven (Abb. 6) in TSHarmem Serum zeigen bei Ablesen auf den dazugehörigen Standardkurven mit zunehmender spezifischer Aktivität nicht nur höhere basale TSH-Werte, sondern (vgl. Tab. 3) auch mit der Menge TSH-Standard zunehmende Differenzen, während diese bei niedrig markiertem ${ }^{125} \mathrm{~J}$-TSH innerhalb der Fehlergrenzen der Methode konstant bleiben.

In Tabelle 3 ist das Verhalten von Standard- zu Wiederfindekurven in Abhängigkeit von der spezifischen Aktivität des eingesetzten ${ }^{125} \mathrm{~J}$-TSH für 12 Assays angegeben. Man erkennt, daß niedrig markierter Tracer (von $8-67 \mathrm{mCi} / \mathrm{mg}$ ) Wiederfindekurven in Humanserum liefert, die entweder mit der Standardkurve identisch sind, oder bei denen die TSH-Differenzen

Tab. 2

Bindung $\left(\frac{B_{n}}{T}\right)$ und Jodid-Abspaltung von verschieden hoch markiertem ${ }^{125} \mathrm{~J}$-TSH

Das markierte TSH wurde verdünnt $(360000 \mathrm{Imp} . / \mathrm{min} \cdot \mathrm{ml})$ und konzentriert $\left(3,8-19,4 \times 10^{\circ} \mathrm{Imp} / \mathrm{min} \cdot \mathrm{ml} \mathrm{bei}-30^{\circ} \mathrm{C}\right.$ eingefroren zwei Wochen gelagert

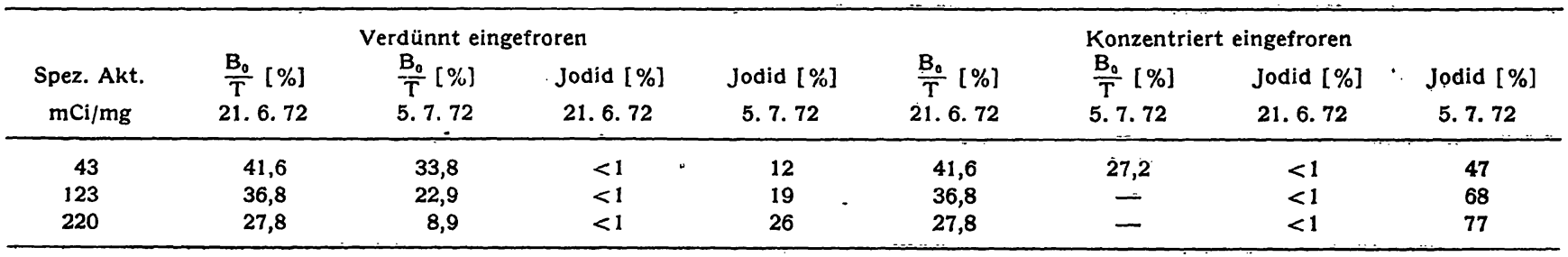




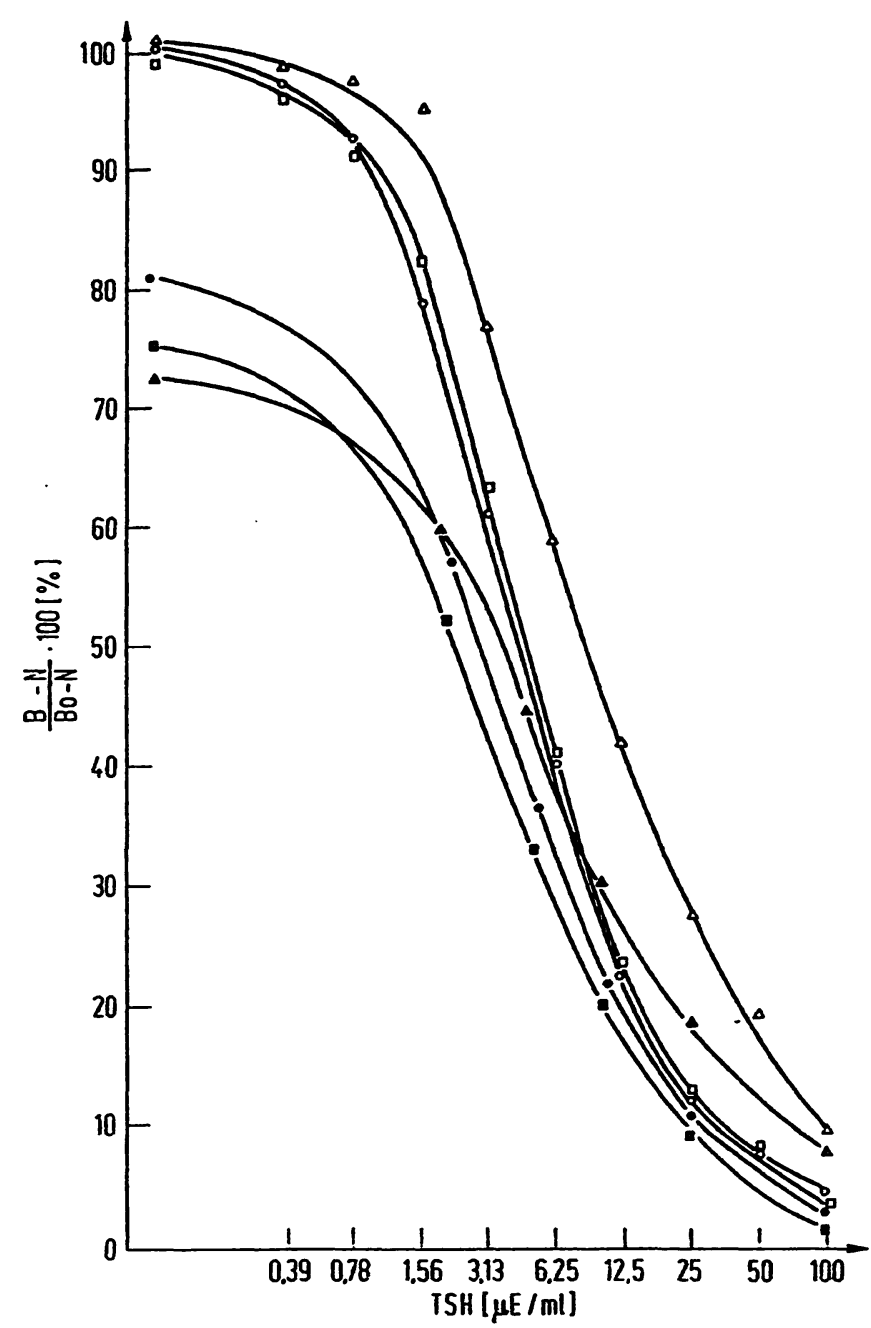

Abb. 6

Standardkurven (offene Symbole) in Puffer B und Wiederfindekurven (geschlossene Symbole) in TSH-armem Serum mit ${ }^{123} \mathrm{~J}-\mathrm{TSH}$ verschie-

(O-O O- O) spez. Akt. $43 \mathrm{mCi} / \mathrm{mg} ; 1700 \mathrm{pg} / 36000 \mathrm{Imp} . / \mathrm{min}$ (므 $\square-\square$ ) spez. Akt. $123 \mathrm{mCi} / \mathrm{mg} ; 600 \mathrm{pg} / 36000 \mathrm{Imp} . / \mathrm{min}$ $(\Delta-\Delta \Delta-\Delta)$ spez. Akt. $\underset{\text { per Probe }}{220} \mathrm{mCi} / \mathrm{mg} ; 340 \mathrm{pg} / 36000 \mathrm{Imp} . / \mathrm{min}$
Tab. 3

Differenzen ( $\Delta \mu \mathrm{E} \mathrm{TSH} / \mathrm{ml}$ ) zwischen Standard- und Wiederfindekurven in Abhăngigkeit von der spezifischen Aktivitāt des Tracers und der Menge TSH-Standard ( ${ }^{\mathrm{E}} \mathrm{E} / \mathrm{ml}, 5$ Meßpunkte)

\begin{tabular}{|c|c|c|c|c|c|}
\hline \multirow{2}{*}{$\begin{array}{c}\text { Spez. Aktivitāt } \\
\text { mCi/mgTSH }\end{array}$} & \multicolumn{5}{|c|}{ TSH-Standard } \\
\hline & $\Delta 0$ & 12 & $\Delta 5$ & $\Delta 10$ & $\Delta 25$ \\
\hline 8 & 0,95 & 1,1 & 0,8 & 0,6 & $-0,5$ \\
\hline 36 & 0,4 & 0,7 & 0,9 & 1,5 & $\mathbf{3 , 5}$ \\
\hline 42 & 0,2 & $-0,1$ & 0,2 & $-0,6$ & 0 \\
\hline 42 & 0,15 & $-0,15$ & 0,9 & 1,0 & 0,7 \\
\hline $43^{*}$ & 1,5 & 1,5 & 2,0 & 2,5 & 2,5 \\
\hline 48 & 0,9 & 0,25 & 0,7 & 3,0 & 3,5 \\
\hline 48 & 0 & $-0,2$ & 0 & 0,1 & 0 \\
\hline 67 & 0,15 & $-0,2$ & $-0,3$ & 0,5 & $-1,2$ \\
\hline 112 & 2,5 & 2,6 & 3,8 & 17 & 18 \\
\hline $123^{*}$ & 2 & 2,4 & 3,4 & 4,2 & 12 \\
\hline $220 *$ & 3,6 & 3,9 & 6,2 & 12 & 20 \\
\hline 254 & 6,6 & 8,5 & 9 & 15 & 30 \\
\hline
\end{tabular}

- Wiederfindekurven mit Tracer verschiedener spezifischer Aktivitāt aus einem Durchgang und einer $\mathrm{Na}^{128} \mathrm{~J}$-Charge.

zwischen Standard- und Wiederfindekurve über den empfindlichen Meßbereich (bis $25 \mu \mathrm{E}$ ) konstant bleiben. Bei höher markiertem ${ }^{125} \mathrm{~J}-\mathrm{TSH}$ (von 112$254 \mathrm{mCi} / \mathrm{mg}$ ) ist die TSH-Differenz bereits größer, entsprechend dem zu hoch gemessenen TSH-Gehalt des Wiederfindeserums und steigt laufend mit zunehmender Menge TSH-Standard an. So werden z. B. bei Anwendung von ${ }^{125} \mathrm{~J}-\mathrm{TSH}$ mit einer spezifischen Aktivitāt von $220 \mathrm{mCi} / \mathrm{mg}$ statt $25 \mu \mathrm{E} / \mathrm{ml} \mathrm{TSH}$ im Wiederfindeserum $45 \mu \mathrm{E} / \mathrm{ml}$ gemessen.

In der Abbildung 7 sind gefundene gegen erwartete TSH-Werte im Wiederfinderersuch als Funktion der spezifischen Aktivität graphisch dargestellt. Nit zunehmender spezifischer Aktivitāt wãchst die Abweichung vom $45^{\circ}$-Winkel.

Qualitātskontrolle durch Wiederfindekurven in Humanserum

In Tabelle 4 wurden 13 TSH-Assays bezüglich spezifischer Aktivität des eingesetzten ${ }^{125} \mathrm{~J}$-TSH und Reproduzierbarkeit $z$ weier in verschiedenem Meß-

Tab. 4

H-TSH-Kontrollwerte über 13 Assays ohne und mit Korrektur durch den Regressionskoeffizienten

\begin{tabular}{|c|c|c|c|c|c|c|}
\hline Assay No & $\begin{array}{l}\text { Spez. Akt. } \\
\text { mCi/mg TSH }\end{array}$ & Regression B & $\mathrm{K}$ niedrig & $\begin{array}{c}\text { K niedrig } \\
\mathrm{B}\end{array}$ & $K$ hoch & $\begin{array}{c}\text { K hoch } \\
\text { B }\end{array}$ \\
\hline 1 & 36 & 1,124 & 4,5 & 4,0 & 26 & 23,1 \\
\hline 2 & 67 & 0,955 & 3,55 & 3,72 & 23,4 & 24,5 \\
\hline 3 & 67 & 0,821 & 3,7 & 4,5 & 19,8 & 24,1 \\
\hline 4 & 48 & 0,896 & 3,05 & 3,4 & 18 & 20,1 \\
\hline 5 & 40 & 1,144 & 4,95 & 3,51 & 24 & 21 \\
\hline 6 & 40 & 1,229 & 4,95 & 3,84 & 24 & 18,5 \\
\hline 7 & 40 & 1,049 & 3,7 & 3,52 & 25 & 23,8 \\
\hline 8 & 40 & 0,983 & 3,7 & 3,76 & 25 & 25,4 \\
\hline 9 & 73 & 1,883 & 6,6 & 3,51 & 40,5 & 21,5 \\
\hline 10 & $120^{*}$ & 1,12 & 4,15 & 3,71 & 23,5 & 21,0 \\
\hline 11 & $300 *$ & 1,278 & 6,15 & 4,8 & 29,8 & 23,2 \\
\hline 12 & 120 & 1,196 & 4,1 & 3,09 & 27 & 22,6 \\
\hline 13 & 300 & 1,658 & 6 & 3,62 & 30,5 & 18,4 \\
\hline $\bar{x}$ & & & 4,6 & 3,77 & 26,13 & 22,1 \\
\hline VK [\%] & & & 24,6 & 11,7 & 22,1 & 9,7 \\
\hline
\end{tabular}

* Ober Discelektrophorese gereinigtes ${ }^{23 \mathrm{~J}} \mathrm{~J}$-TSH. 


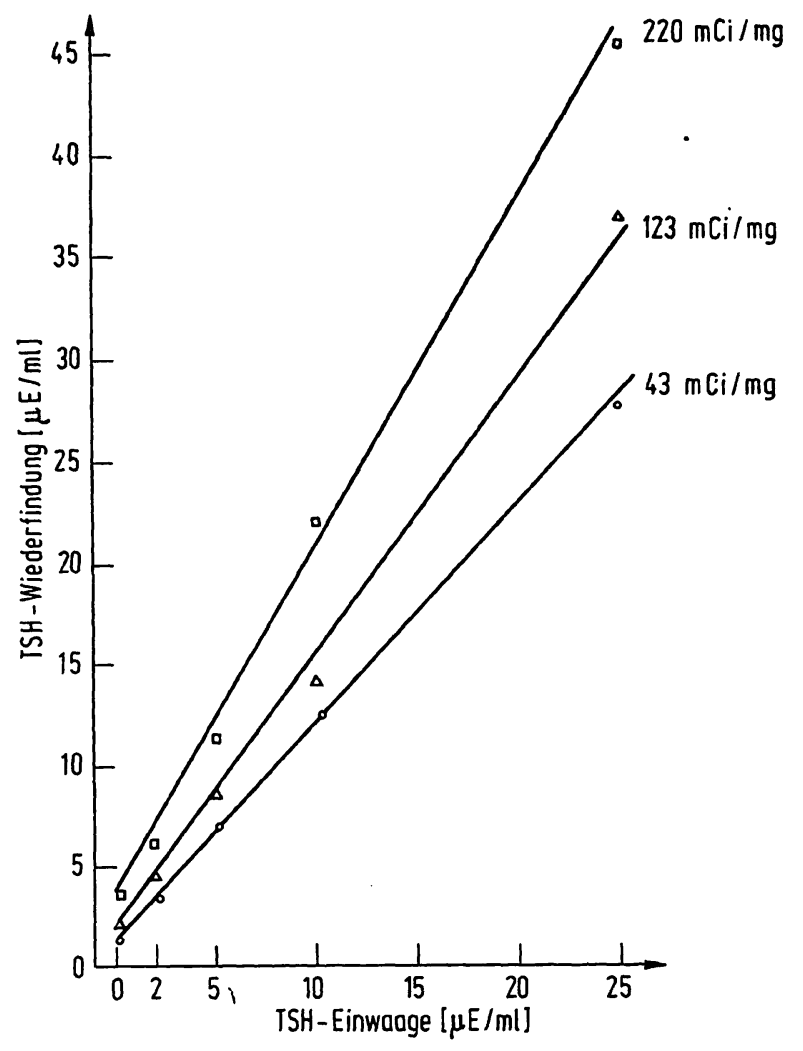

Abb. 7

TSH-Wiederfindung in Humanserum in Abhängigkeit von der spezifischen Aktivität des ${ }^{125} \mathrm{~J}-\mathrm{TS} \mathrm{H}$

bereich gelegener Kontrollwerte verglichen. Weiter wurde für jeden Durchgang der Regressionskoeffizient für die Gerade, die bei Auftragen von Standard- gegen Wiederfindekurve (Abb. 7) resultiert, berechnet.

Die Kontrollwerte wurden mit diesem Regressionskoeffizienten korrigiert und schließlich die Variationskoeffizienten der Kontrollwerte vor und nach dieser Korrektur verglichen.

Die Regressionskoeffizienten schwanken zwischen 0,82 und 1,88, wobei im Mittel bei Tracer mit spezifischer Aktivität über $100 \mathrm{mCi} / \mathrm{mg}$ TSH $30 \%$ zu hohe TSHWerte gemessen werden. Die Interassay-Variationskoeffizienten der Kontrollwerte sinken im Mittel nach der Korrektur mit Hilfe des Regressionskoeffizienten für jeden Durchgang um mehr als einen Faktor von 2.

\section{Diskussion}

Die Kinetik der Bindung von ${ }^{125} \mathrm{~J}-\mathrm{TSH}$ an den TSHspezifischen Antikörper (Abb. 1) bei verschiedenen Temperaturen zeigt, daß Raumtemperatur $\left(20^{\circ} \mathrm{C}\right)$ sowohl bezüglich der Geschwindigkeit der Gleichgewichtseinstellung, als auch im Hinblick auf die Höhe der Bindung $\left(\frac{B_{0}}{T}\right)$ allen anderen Temperaturen überlegen ist. Bei $20^{\circ} \mathrm{C}$ wird offenbar ein Optimum zwischen Reaktions- und Dissoziationsgeschwindigkeit von Antigen und Antikörper erreicht. Bei $4^{\circ} \mathrm{C}$ ist das „Plateau“ selbst nach sechstägiger Inkubation noch wesentlich steiler als bei allen anderen Temperaturen und das Gleichgewicht wird nicht erreicht. Bei Temperaturen über $20^{\circ} \mathrm{C}$ überwiegt die Dissoziation zunehmend. Das führt dazu, daß bei $37^{\circ} \mathrm{C}$ das „Plateau“ zwar schon nach etwa $50 \mathrm{~h}$ erreicht wird, aber eine etwa $15 \%$ niedrigere Bindung als bei $20^{\circ} \mathrm{C}$ resultiert (Abb. 1).

Die Kinetik der Fällungsreaktion mit dem 2. Antikörper (Abb. 2) bei Raumtemperatur zeigt, daß es sich hier um eine rascher verlaufende Reaktion handelt. Die je nach verwendeter Anti- $\gamma$-Globulin-Präparation gefundenen Unterschiede machen es erforderlich, die optimale Verdünnung und die Kinetik für jede neue Charge zu prüfen und eventuell das angegebene Inkubations-Schema (Tab. 1) zu adaptieren. Wir sehen keinen Grund dafür, die Präzipitationsreaktion prinzipiell „klassisch“, d. h. bei $4^{\circ} \mathrm{C}$ über 12 oder mehr Stunden ablaufen zu lassen (Abb. 3).

Die Optimierungsversuche in Abbildung 3 zeigen für jede Kaninchenserum-Konzentration eine optimale Antikörper-Konzentration, bei der die maximale Fällung erfolgt. Eine Erhöhung der Antikörper-Konzentration führt $\mathrm{zu}$ einer Abnahme des radioaktiven Präzipitats. Wir führen diesen Effekt auf die Polyvalenz der präzipitierenden Antikörper zurück und auf die Notwendigkeit, mehr àls ein $\gamma$-Globulin-Molekül per Anti- $\gamma$-Globulin binden zu müssen, damit der AntigenAntikörper-Komplex ausfällt.

Die Vorinkubation von 1. Antikörper mit kaltem Hormon ist in bezug auf die Empfindlichkeit der Methode (untere Nachweisgrenze) allen übrigen geprüften Inkubationsbedingungen überlegen (Abb. 4). Durch längere Vorinkubation kann die untere Nachweisgrenze weiter herabgesetzt werden. Wenn wir mit dieser Feststellung im Gegensatz $z u$ anderen Autoren stehen (5), so möglicherweise deshalb, weil von diesen bei $4^{\circ} \mathrm{C}$ und nicht bei der optimalen Temperatur von $20^{\circ} \mathrm{C}$ mit einem ${ }^{125} \mathrm{~J}$-TSH-Tracer gearbeitet wurde, der in der Regel mit einer spezifischen Aktivität von mehr als $100 \mathrm{mCi} / \mathrm{mg}$ nach unseren Erfahrungen übermarkiert ist.

Mit einer zu hohen Markierung nimmt die immunologische Aktivität von ${ }^{125} \mathrm{~J}$-TSH gegenüber nicht markiertem H-TSH ab. Nach unseren Ergebnissen ist es optimal, einen ${ }^{125} \mathrm{~J}$-TSH-Tracer zu verwenden, der mit etwa $20-70 \mathrm{mCi} / \mathrm{mg}$ nach „klassischen“ Vorstellungen niedrig markiert ist. Niedrig markiertes ${ }^{125} \mathrm{~J}-\mathrm{TSH}$ ist erstens stabiler gegenüber strahlenchemischer Zersetzung und wird zweitens trotz mehrfach höherer Konzentration per Probe besser vom Antikörper gebunden. Dieser Effekt tritt noch deutlicher in Erscheinung; wenn man nicht die Impulsrate, soridern die Tracermenge konstant hält. Drittens liefert niedrig markierter Tracer gleich empfindliche Standardkurven und ergibt viertens richtigere TSH-Werte als ${ }^{125} \mathrm{~J}-\mathrm{TSH}$, das mit mehr als $100 \mathrm{mCi} / \mathrm{mg}$ markiert ist. Erst bei ganz niedrigen spezifischen Aktivitäten, wie z. B. $8 \mathrm{mCi} / \mathrm{mg}$, sinkt, wie zu erwarten, die Empfindlichkeit der TSHBestimmung wegen der hohen Tracer-Konzentration im Assay ab (50\% Intercept $45 \mu \mathrm{E} \mathrm{TSH} / \mathrm{ml}$ gegenüber $6-9 \mu \mathrm{E} \mathrm{TSH} / \mathrm{ml}$ bei $20-70 \mathrm{mCi} / \mathrm{mg}$ ) allerdings ohne Verlust der Richtigkeit (Tab. 3), da die Wiederfindekurve mit der Eichkurve nahezu identisch verläuft. Wir kommen ferner zu der Erkenntnis, daß es zu- 
mindest bei ${ }^{125} \mathrm{~J}$-TSH günstiger ist, sofort nach der Markierung in Gebrauchskonzentration (pro $\mathrm{ml}$ etwa $250000 \mathrm{Imp} . / \mathrm{min}$ ) und tiefgefroren aufzubewahren, als konzentriert einzufrieren und jedesmal vor Gebrauch nachzureinigen.

Die bessere relative Richtigkeit, $d . h$. in bezug auf den verwendeten Standard, wird bewiesen durch die Konstanz der Differenzen zwischen Standard und Wiederfindekurven (Tab. 3) über den empfindlichen Meßbereich. Die in der Literatur $(1,5,7)$ häufig beschriebenen Differenzen zwischen TSH-Werten in Pufferlösungen mit Rinderalbuminzusatz und in $\mathrm{Hu}-$ manserum finden ihre Erklärung durch die Verwendung von zu hoch markiertem TSH. Sie treten entweder gar nicht oder viel schwächer bei Verwendung von TSH-Tracer geringerer spezifischer Aktivität auf.

Das Mitführen von Wiederfindekurven in gepooltem oder supprimiertem Humanserum in jedem Assay gestattet nicht nur den systematischen Fehler besser zu erfassen, als dies durch Kontrollseren allein möglich ist, sondern zudem die Korrektur des gesamten Assays. Dies kommt klar zum Ausdruck durch die Verbesserung des Interassay-Variationskoeffizienten für die Kontrollseren. Die Tabelle 4 zeigt zudem, daß der mittlere Regressionskoeffizient für niedrig markierten Tracer $(36-67 \mathrm{mCi} / \mathrm{mg})$ mit $1,025 \mathrm{dem}$ Idealwert von 1,0 sehr nahekommt.

Aufgrund der hier mitgeteilten methodischen Verbesserungen liegt der Normalbereich der basalen TSHSpiegel $(\overline{\mathrm{x}} \pm 2 \mathrm{~s})$ jetzt $z$ wischen 0 und $3,8 \mu \mathrm{E} / \mathrm{ml}$ und damit niedriger als der früher mitgeteilte Bereich von 1-7 $\mu \mathrm{E} / \mathrm{ml}$ (8). Der Anstieg der TSH-Spiegel nach Stimulation von Kontrollpersonen mit $200 \mu \mathrm{g}$ TRH i. v. blieb mit einem $\Delta \mathrm{TSH}_{30 \text { min }}$ von $2,7-23,6 \mu \mathrm{E} / \mathrm{ml}$ $\left(\overline{\mathbf{x}}_{\log } \pm 2 \mathrm{~s}\right)$ praktisch gleich $(8)$.

\section{Literatur}

1. Utiger, R. D., Odell, W. D. \& Condliff, P. G. (1963), Endocr. 73, 359-365. - 2. Golstern-Golaire, J. \& VANHAELST, L. (1970), Internat. J. Appl. Rảdiat. Isotop. 21, 17-20. - 3. PATEL, Y. C., Burger, H. G. \& Hudson, B. (1971), J. Clin. Endocr. 33, 768-774. - 4. Hall, R., Amos, J. \& Ormston, B. J. (1971), Brit. Med. J. 1, 582-585. - 5. vON zUR MÜHLEN, A. \& EMRICH, D. (1971), diese Z. 9, 257-265. - 6. GreENWOOD, F. C., HuNTER,
W. M. \& Glover, J. (1963), Biochem. J. 89, 114-123. - 7. Odell, W. D., Vastager, L. \& Bates, R. (1968), Radioimmunoassay of human thyrotrapin. In: Radioisotopes in medicine; in-vitro studies. US Atomic Energy Commission. - 8. PrCKardr, C. R., Erhardt, F., Grüner, J. Horn, K. \& SCriba, P. C. (1972), Klin. Wochenschr. 50, 1134-1137.
Prof. Dr. P. C. Scriba 8000 München 2 Ziemssenstr. 1 